\title{
Genome sequencing of high-penicillin producing industrial strain of Penicillium chrysogenum
} \author{
Jun $\mathrm{Yu}^{2}$, Jiayan $\mathrm{Wu}^{2^{*}}$, Baoling Duan ${ }^{1^{*}}$ \\ From The Twelfth Asia Pacific Bioinformatics Conference (APBC 2014) \\ Shanghai, China. 17-19 January 2014
}

Fu-Qiang Wang ${ }^{1 \dagger}$, Jun Zhong ${ }^{2,3 \dagger}$, Ying Zhao', Jingfa Xiao², Jing Liu' Meng Dai', Guizhen Zheng ${ }^{1}$, Li Zhang ${ }^{1}$,

\begin{abstract}
Background: Due to the importance of Penicillium chrysogenum holding in medicine, the genome of low-penicillin producing laboratorial strain Wisconsin54-1255 had been sequenced and fully annotated. Through classical mutagenesis of Wisconsin54-1255, product titers and productivities of penicillin have dramatically increased, but what underlying genome structural variations is still little known. Therefore, genome sequencing of a highpenicillin producing industrial strain is very meaningful.

Results: To reveal more insights into the genome structural variations of high-penicillin producing strain, we sequenced an industrial strain P. chrysogenum NCPC10086. By whole genome comparative analysis, we observed a large number of mutations, insertions and deletions, and structural variations. There are 69 new genes that not exist in the genome sequence of Wisconsin54-1255 and some of them are involved in energy metabolism, nitrogen metabolism and glutathione metabolism. Most importantly, we discovered a $53.7 \mathrm{~Kb}$ "new shift fragment" in a seven copies of determinative penicillin biosynthesis cluster in NCPC10086 and the arrangement type of amplified region is unique. Moreover, we presented two large-scale translocations in NCPC10086, containing genes involved energy, nitrogen metabolism and peroxysome pathway. At last, we found some non-synonymous mutations in the genes participating in homogentisate pathway or working as regulators of penicillin biosynthesis.

Conclusions: We provided the first high-quality genome sequence of industrial high-penicillin strain of $P$. chrysogenum and carried out a comparative genome analysis with a low-producing experimental strain. The genomic variations we discovered are related with energy metabolism, nitrogen metabolism and so on. These findings demonstrate the potential information for insights into the high-penicillin yielding mechanism and metabolic engineering in the future.
\end{abstract}

\section{Background}

Penicillin and $\beta$-lactam antibiotic play a significant role in human medical history [1,2] since Fleming's discovery of the filamentous fungus Penicillium notatum in 1929 [3]. The regulation of penicillin biosynthesis has been studied

\footnotetext{
* Correspondence: 18603113322@163.com; wujy@big.ac.cn

† Contributed equally

${ }^{1}$ New Drug Research and Development Center of North China

Pharmaceutical Group Corporation, National Engineering Research Center of Microbial Medicine, Hebei Industry Microbial Metabolic Engineering \&

Technology Research Center, Shijiazhuang, Hebei 050015, China

${ }^{2}$ CAS Key Laboratory of Genome Sciences and Information, Beijing Institute

of Genomics, Chinese Academy of Sciences, Beijing 100101, China
}

Full list of author information is available at the end of the article for many years, together with much more proteins or pathways were discovered [4-9]. The improvement of $P$. chrysogenum strains to obtain higher penicillin yields is a main intense objective in industrial research $[10,11]$.

Due to the importance of $P$. chrysogenum, the genome sequence of low-penicillin producer Wisconsin54-1255, which is widely used in laboratories, was sequenced and a number of genes responsible for key steps in penicillin production were identified [12]. The precursors for penicillin biosynthesis, genes encoding microbody proteins and transporters were found, illustrating potential for future genomics-driven metabolic engineering [12]. Through classical mutagenesis and screening methods, product 
titers and productivities of penicillin have dramatically increased since Wisconsin54-1255 strain, but how lowpenicillin producer strain was transformed into an efficient producer through improvement is still challenging. For commercial reasons, the improvement of $P$. chrysogenum strains has never been stopped. The productivity of industrial used strains is far more higher than their ancestor, and the progress was mainly obtained by classical mutagenesis and screening methods. Because mutations were random, most of the genetic changes in high yield strains were unclear. Although some significant structural variations (SVs) $[8,9,13]$ and differential expression profiling $[12,14,15]$ have been found in high-penicillin producing strains, little is known about the underlying whole genomic changes between low-producing laboratorial strain and high-producing industrial strain.

To gain more insight into the genome structural variations of high-penicillin producing strain, we sequenced a Chinese industrial strain NCPC10086. We also offer a comprehensive comparative genomics analysis [16-19] to find all mutations and large-scale structural variations between NCPC10086 and the first published genome of P. chrysogenum strain, Wisconsin54-1255 [12]. Some variations including mutations, indels and structural variations were considered for their potential biological impact for penicillin biosynthesis. Our genome sequence data and analyses explore the differences between highand low-yield $P$. chrysogenum strains and demonstrate the potential useful information to improve strains by direct genetic engineering tools.

\section{Results}

Genome sequencing, assembly and general characeristics We sequenced the genome of $P$. chrysogenum NCPC10086 using a whole-genome shotgun sequencing strategy $[20,21]$. Owing to different sequencing technologies have various advantages and disadvantages $[22,23]$, we generated a high quality genome assembly using a combination of first and second generation sequencing platforms and strategies (Table 1). First, we generated single-end reads using Roche 454 pyrosequencing platform [24] and matepair reads with 3-4 $\mathrm{Kb}$ and 6-8 $\mathrm{Kb}$ insert fragment sizes, using ABI 3730 and MegaBACE 1000 Sanger sequencing platforms [25], respectively. Then we generated mate-pair reads with $1-2 \mathrm{~Kb}$ insert fragment size using Illumina HiSeq 2000 sequencing platform [26,27] and used all mate-pair reads to join contigs into scaffolds. Overall, we get $204 \times$ sequencing coverage of high quality reads for de novo assembly (Table 1 ).

We got a total genome size of $32.3 \mathrm{Mb}$ (Table 2) similar as Wisconsin54-1255 [12]. The length of longest contig is $1,655 \mathrm{~Kb}$, which indicates fine continuity of assembly. Owing to the deeper sequencing data, the contig N50 of NCPC10086 is $661 \mathrm{~Kb}$ which is 70\% higher than Wiscon$\sin 54-1255(389 \mathrm{~Kb})$. The scaffold N50 of our genome is $2.8 \mathrm{Mb}$ and the longest scaffold is $4,063 \mathrm{~Kb}$, illustrating our genome is suitable for structural variations detection, especially large-scale translocations. The gene structures were predicted with a combined de novo and homology-based approach. Firstly, we masked all the repeat sequences in the genome and used Fgenesh (v 3.1.2)[28] and GeneMarkES (v 2.3e)[29] to provide an initial set of 11,284 predicted ORFs. Secondly, we took advantage of the gene prediction results of Wisconsin54-1255 [12] to revise and complement our predicted genes by homology searches. At last, the former two results were integrated together, and 13,290 protein-coding genes were predicted in P. chrysogenum NCPC10086 genome (Table 2). GC content of the genome is $48.9 \%$ and every 2,430 bp has one gene. The mean gene length is $1,499 \mathrm{bp}$ and most of the genes have introns.

As to gene annotation, four databases of Non-redundant (NR), InterProscan [30,31], Swiss-Prot/UniProtKB $[32,33]$ and Gene Ontology system (GO) $[34,35]$ were used to annotate 13,290 predicted genes of NCPC10086 (Figure 1A). We found 12,906 genes have homolog in NR, 9,371 genes have protein structural domains in InterProscan, and 7,625 genes have homolog in SwissProt/UniProtKB, and 6,831 genes can be classified in GO. There are 6,140 genes can be annotated by all four gene annotation systems (Figure 1A), suggesting these genes are well studied. The second large genes group is NR-specific with the number of 3,510, which indicates functions of these genes are just beginning to be understood. The following two large groups are 1,362 genes with homolog in Swiss-Prot database and 1,081 genes

Table $1 P$. chrysogenum NCPC10086 genome sequencing data

\begin{tabular}{lcccc}
\hline Instruments & Insert fragment size $\mathbf{( K b )}$ & $\begin{array}{c}\text { Reads length } \\
(\mathbf{b p})\end{array}$ & Sequencing throughput (Mb) & Coverage \\
\hline Roche 454 GS & single end & 410 & 614 & $18 \times$ \\
Illumina HiSeq 2000 & $1-2$ & 50 & 6,120 & $180 \times$ \\
ABI 3730 & $3-4$ & 659 & 170 & $5 \times$ \\
MegaBACE 1000 & $6-8$ & 739 & 34 & $1 \times$ \\
Total & - & - & 6,938 & $204 \times$ \\
\hline
\end{tabular}

The estimated genome size of $P$. chrysogenum NCPC10086 is about $34 \mathrm{Mb}$. 
Table 2 Global statistics of the genome assembly and annotation of $\boldsymbol{P}$. chrysogenum NCPC10086

\begin{tabular}{lccccc}
\hline Assembly & Number & N50 (Kb) & Longest (Kb) & Size (Mb) & Percentage of the assembly \\
\hline Contigs & 327 & 661 & 1,655 & 32.2 & 99.7 \\
Scaffolds & 175 & 2,847 & 4,063 & 32.3 & 100 \\
\hline Annotation & Number & Mean length & GC content (\%) & $\begin{array}{c}\text { Gene density } \\
\text { (1 gene every } \mathbf{~ b p ) ~}\end{array}$ \\
\hline Coding genes & 13,290 & 1,499 & 48.9 & 2,430 \\
Genes with intron & 10,966 & 1,559 & 51.6 & 2,945 & \\
\hline
\end{tabular}
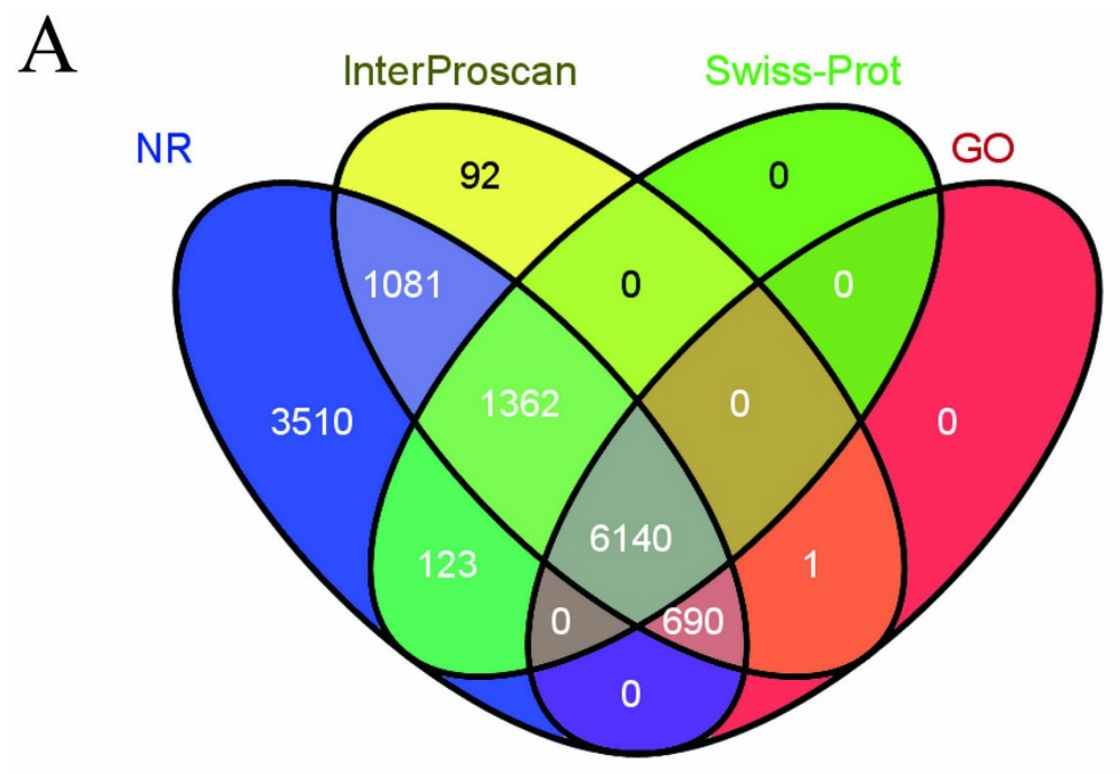

B
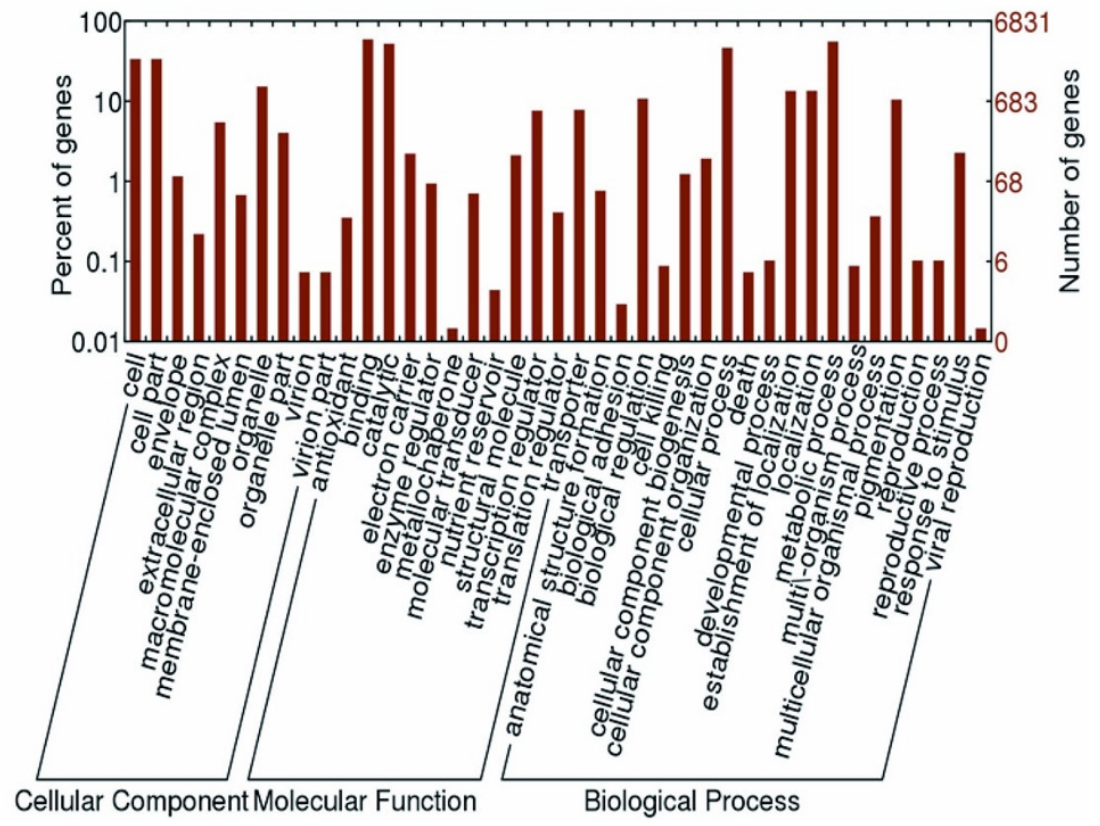

Figure 1 Gene annotation and gene ontology of $P$. chrysogenum NCPC10086. (A) Venn diagram showing unique and shared proteins could be annotated by databases of Non-redundant, InterProscan, Swiss-Prot/UniProtKB and Gene Ontology. (B) There are 6,831 proteins could be assigned to cellular component, biological process and molecular function by Gene Ontology classification system. 
with protein domain in InterProscan. Both of them cannot be assigned to GO system, indicating functions of these genes are little known but ready for deeper investigation in some extent. There are 6,831 genes can be assigned at least one $\mathrm{GO}$ term for describing cellular component, biological process and molecular function classification (Figure 1B).

\section{Genome comparison analysis between $P$. chrysogenum NCPC10086 and Wisconsin54-1255}

P. chrysogenum Wisconsin54-1255 is a low-penicillin producer strain widely used in libraries. The genome sequence of Wisconsin54 was well sequenced in 2008, which is the first published genome of $P$. chrysogenum [12]. The evolutionary relationship between Wisconsin54-1255 and our sequenced high-penicillin strain NCPC10086 is very close. Here we offer a comprehensive genome comparison analysis between these two $P$. chrysogenum strains, and try to figure out some interesting genomics discrepancy. To access comparative genomics statistics results, we aligned all the genes of Wisconsin54-1255 to the scaffolds of NCPC10086 to detect gene mutations. There are 12,943 predicted genes in Wisconsin54-1255 and 13,290 predicted genes in NCPC10086. And we discovered 11,573 genes are identical between two strains, $89 \%$ for Wisconsin 54 and $87 \%$ for NCPC10086, which indicates these two genomes are very conservative (Figure $2 \mathrm{~A}$ ). As to the non-identical genes, 1,154 genes' identity are higher than $90 \%$, part of them leading to mutations, and only 22 genes have less than $60 \%$ identity. In addition, we complemented 64 genes, which are partial in Wisconsin54-1255 and 514 noncoding sequence regions are redefined as proteincoding genes. Using Wisconsin54-1255 genome sequence as a reference, we realigned $50 \times$ high-quality short reads of NCPC10086 from Illumina HiSeq 2000 to identify single nuclear variations (SNVs). In order to differentiate sequencing errors from mutations, we used three thresholds to filter out unreliable mutation results: (1) we required at least five reads for each mutation; (2) average quality of each mutation had to be higher than 20; (3) there had to be at least one pair of mate-pair reads to support. We got 759 SNVs in coding regions and 1,813 SNVs in noncoding regions. There are 135 SNVs in intron regions, 177 are synonymous mutations and 447 are nonsynonymous mutations, including 34 termination codon mutations. All SNVs result in coding region is described as Figure 2B. Furthermore, we found 35 deletions and 19 insertions in exon regions, 86 copy number variations (CNVs) with the total size of $176,684 \mathrm{bp}$. The polymorphic genes with non-synonymous mutations are listed in Additional file 1.

Besides, we found 69 new genes that do not exist in the available genome sequence of Wisconsin54-1255 [12]. We

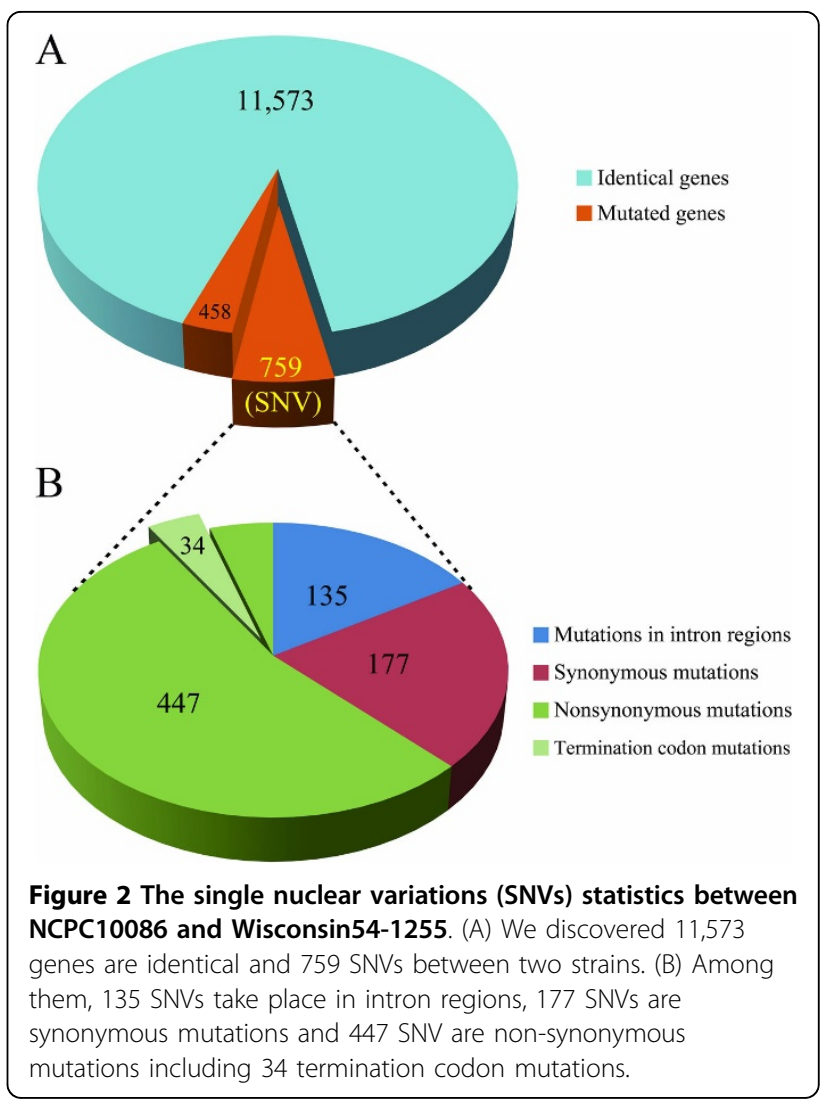

analyzed these new genes carefully and figured out several metabolism, biosynthesis and degradation (Table 3). Firstly, Pch125g10680, Pch106g00010, Pch114g00050 genes involved in amino sugar, nucleotide sugar metabolism, N-Glycan biosynthesis and oxidative phosphorylation may provide more energy in high-penicillin producer for penicillin synthesis. Secondly, we found another new gene, Pch106g00010, involved in nitrogen metabolism, which is up regulated strongly in cultures supplemented with the side chain precursor PAA (phenylacetic acid) in highproducing strain [36]. The last but not the least, the new gene, Pch018g00010, was discovered to participate in glutathione metabolism that may boost production of penicillin. The biosynthesis of cysteine is precursor for the penicillin biosynthesis, and the genes involved in this pathway were over expressed in the high-penicillin producing strain [12]. As to the previous study [37], the increase in the cysteine biosynthesis requires a large NADPH supply, but the oxidized glutathione under oxidative stress also requires NADPH, which could reduce the cysteine biosynthesis. So, we presume that glutathione metabolism may save NADPH and indirectly promote the penicillin production.

To our best knowledge, the penicillin biosynthetic genes cluster (hereafter named PBC) located at chromosome I in P. chrysogenum is the dominant core for 
Table 3 Metabolism or progress involved by several "new" genes

\begin{tabular}{|c|c|c|c|}
\hline Gene name & $\begin{array}{l}\text { Length } \\
\text { (bp) }\end{array}$ & $\begin{array}{l}\text { Location } \\
\text { (bp) }\end{array}$ & The metabolism or progress \\
\hline Pch125g10680 & 4980 & $\begin{array}{c}\text { scaf125 } \\
(3,231,484-3,236,463)\end{array}$ & Amino sugar and nucleotide sugar metabolism \\
\hline Pch106g00010 & 769 & $\begin{array}{c}\text { scaf106 } \\
(193-961)\end{array}$ & Nitrogen metabolism, oxidative phosphorylation \\
\hline Pch114g00050 & 153 & $\begin{array}{c}\text { scaf1 14 } \\
(16,204-16,356)\end{array}$ & Oxidative phosphorylation \\
\hline Pch041g00010 & 713 & $\begin{array}{c}\text { scaf041 } \\
(112-824)\end{array}$ & Riboflavin metabolism \\
\hline Pch056g00010 & 787 & $\begin{array}{l}\text { scaf056 } \\
(36-822)\end{array}$ & $\mathrm{N}$-Glycan biosynthesis \\
\hline Pch018g00010 & 694 & $\begin{array}{c}\text { scaf018 } \\
(325-1,018)\end{array}$ & Glutathione, arachidonic acid, taurine and hypotaurine metabolism, \\
\hline Pch180g00010 & 580 & $\begin{array}{l}\text { scaf180 } \\
(50-629)\end{array}$ & Fluorobenzoate, chlorocyclohexane and chlorobenzene, toluene degradation \\
\hline
\end{tabular}

penicillin production $[9,38]$, which exist in all strains, including NRRL 1951 (wild-type) [39], Wisconsin54-1255, NCPC10086, P2 (Panlabs), AS-P-78 and E1 (Antibioticos, S.A.) [13]. Three penicillin biosynthetic genes, $p c b A B$, $p c b C$ and $p e n D E$ are gathered in this cluster (Figure $3 \mathrm{~A}$ ). Copy number and fragment arrangement are key features about $\mathrm{PBC}$, which could impact on the yield of penicillin. $\mathrm{PBC}$ was amplified five to sixteen copies in different highpenicillin producers, such as five or six copies for AS-P-78 and twelve to fourteen copies for E1 [13] (Figure 3B). As to the low-productive strain Wisconsin54-1255, PBC just has one single copy with the length of $56.9 \mathrm{~Kb}$, consisting of a $53.7 \mathrm{~Kb}$ fragment and a $3.2 \mathrm{~Kb}$ shift fragment bounded by a conserved TGTAAA/T hexanucleotide [8] (Figure 3A). Through reads coverage detection method, we found seven copies of PBC in NCPC10086 with the length of $56.9 \mathrm{~Kb}$, including two copies for 110.6 Kb fragment of D-EG'-F'E'-D' and 56.9 Kb fragment of E'-D'F-G, and one copy for $56.9 \mathrm{~Kb}$ fragment of E'-D'G'-F' (Figure 3B). Compared with the previous investigations $[8,9,13,40]$, one $\mathrm{PBC}$ fragment arrangement in NCPC10086 is unique and has never been reported before, orange bar and blue letters shown in Figure 3B. It is a $53.7 \mathrm{~Kb}$ "NEW SHIFT FRAGMENT" in our genome. We believe the TTTACA hexanucleotide and its inverse complement TGTAAA could be hot spots for site-specific recombination after mutation with nitrosoguanidine. Unfortunately, the length of PBC is so long that we cannot get the full precise arrangement of these copies.

The genes involved in fungal secondary metabolic pathways share a common tendency towards physical cluster, with a preference for subtelomeric regions [41]. The wide range of translocation is a very interesting phenomenon happened in industrial filamentous fungus, for example, Aspergillus niger [16]. Comparison with Wisconsin54-1255, we found two large fragment translocations in NCPC10086. One is a $266 \mathrm{~Kb}$ fragment in subtelomere (position 5,739,429-6,005,263 in scaffold22 of Wisconsin54-1255) transferred to the around centromere in NCPC10086 and the range of translocation is about $3 \mathrm{Mb}$ (Figure 4A). To avoid the possibility of assembly error, we realigned mate-pair reads around the breakpoint of translocation and found enough reads to across the breakpoint (Figure 4B). Moreover, we did PCR identification around the breakpoint and only NCPC (N) has band with estimated size (Figure 4C). This $266 \mathrm{~Kb}$ fragment of translocation includes 107 genes from Pc22g24360 to Pc22g25450 and their mean genes size is 1,638 bp. Unfortunately, the functions of most genes are unknown except for Pc22g24480 (nre), which encodes a regulator of nitrogen metabolite repression (red boxed in Figure 4A). We hypothesize that the translocation of Pc22g24480 may promote the nitrogen metabolism in NCPC10086, corresponding to the "new" gene discovered in nitrogen metabolism, which is described earlier in this article. Another translocation is a $1,202 \mathrm{~Kb}$ fragment (position 387,513$1,589,522$ bp in scaffold18 of Wisconsin54-1255) transferred to scaffold072 of NCPC10086 starting at position $148,434 \mathrm{bp}$ (Figure 5A). The 1,202 $\mathrm{Kb}$ fragment consists of 494 genes from Pc18g01610 to Pc18g06720 and their mean size is $1,480 \mathrm{bp}$. Those genes associate with energy metabolism and peroxisome pathway, such as Pc18g02420, which encodes mitochondrial ADP/ATP carrier, and Pc18g02550, which encodes PEX-2 (red boxed in Figure 5A). We also did mate-pair reads alignment around the breakpoint of translocation (Figure 5B) and PCR identification (Figure 5C) to certificate this translocation.

At last, we focused on some genes involved in homogentisate and the regulators of penicillin biosynthesis. Table 4 shows the comparison results of these genes between Wisconsin54-1255 and NCPC10086. PahA gene encodes a phenylacetate 2-hydroxylase that catalyzes the first step of phenylacetate catabolism, decreasing the precursor availability for penicillin biosynthesis [42]. Owing 


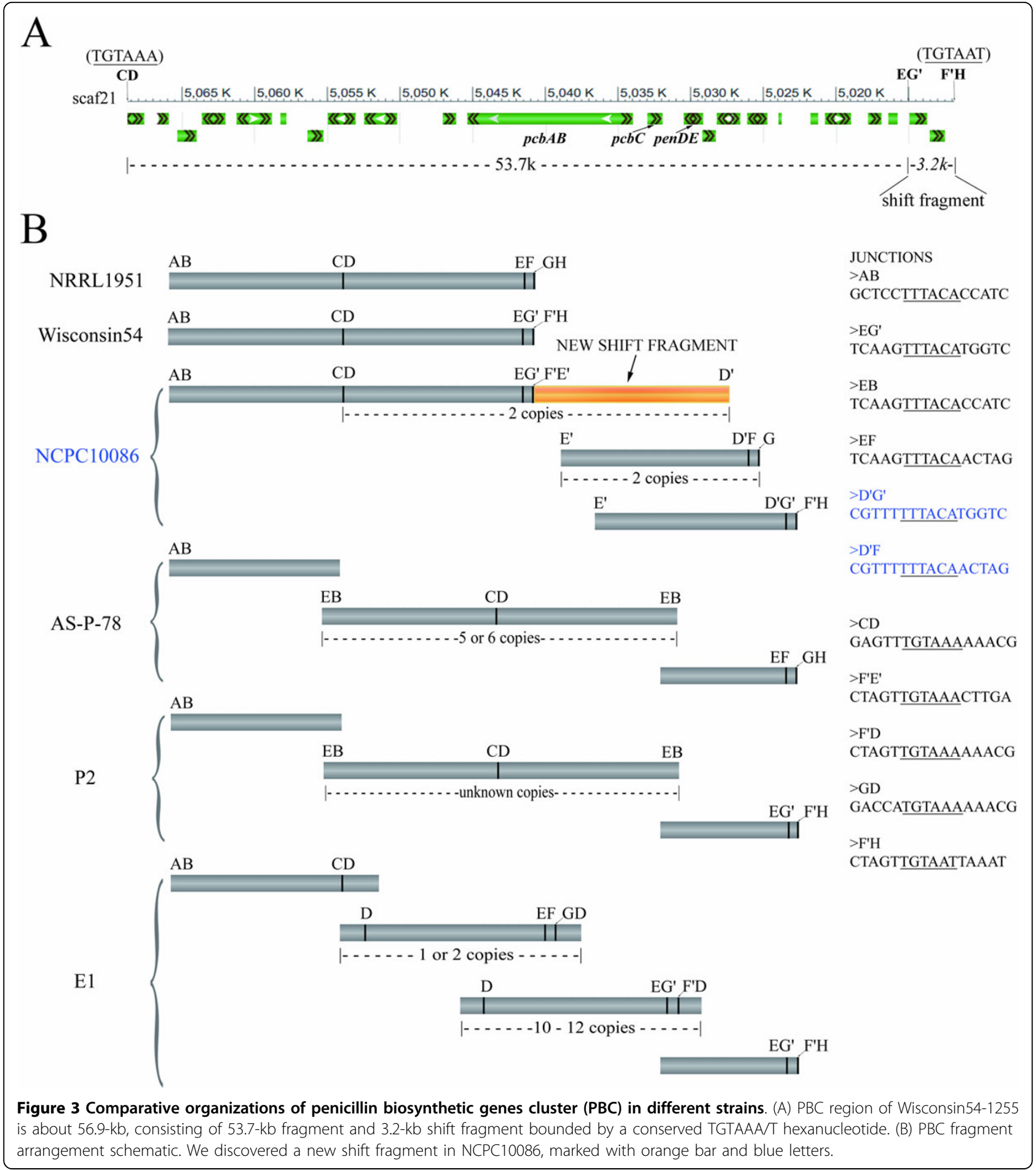

to a point mutation $\left(\mathrm{C}^{598} \rightarrow \mathrm{T}\right)$ in pahA gene, the homogentisate pathway for PAA catabolism has been reported to be largely inactivated but the penicillin yield is increased in Wisconsin54-1255 [42]. Based on the comparative genomics analysis, we found pahA gene was shown in a $27.1 \mathrm{~Kb}$ translocation, but pahA gene is identical between the two strains. Three other genes, pahB (Pc22g02230), pahC (Pc16g01770) and pahD (Pc21g22560), are strongly similar to pahA and all of them belonging to cytochrome $\mathrm{P} 450$ monooxygenases. For pahC gene, there is a mutation $\left(\mathrm{G}^{482} \rightarrow \mathrm{C}\right)$ causes a single amino-acid substitution at position 129 of the 


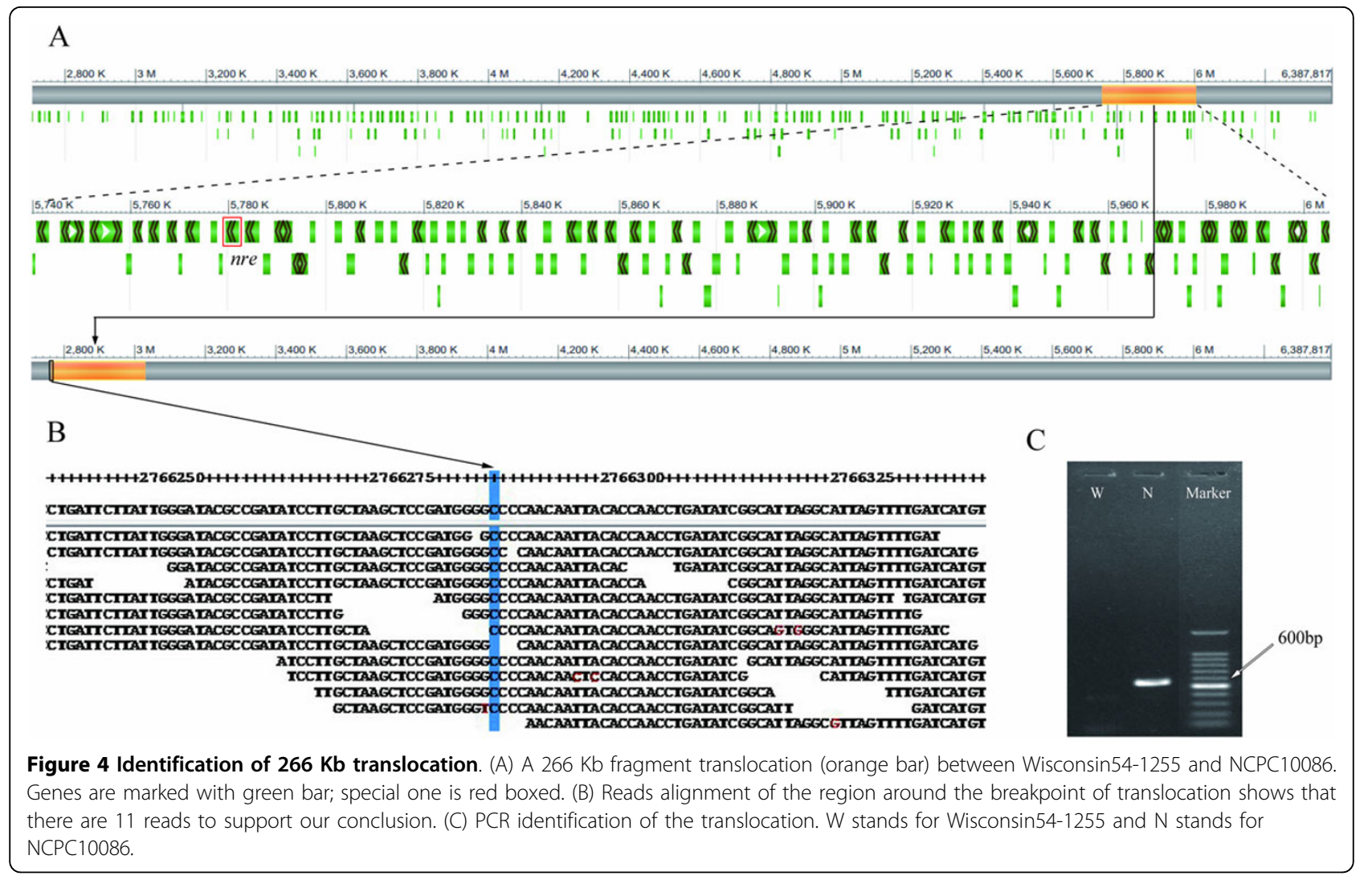

protein: an alanine residue in strain Wisconsin54-1255 has been substituted to proline in NCPC10086 strain (A129P). For pahD gene, we found a synonymous mutation $\left(\mathrm{C}^{1976} \rightarrow \mathrm{T}\right)$. All the promoter sequences of these genes are all the same. PclaeA gene was found as a global regulator of secondary metabolism, including penicillin biosynthesis, sporulation and pigmentation in P. chrysogenum [43], which is identical in sequence in the genome of Wisconsin54-1255 and NCPC10086 with the same copy number. Due to PclaeA gene encodes a velvet-like complex, we checked another velvet-like complex PcVelA. Its corresponding PcvelA gene is a key regulator of metabolism, acting both as an activator and repressor of secondary metabolism in P. chrysogenum [44]. There is a mutation $\left(\mathrm{C}^{1002} \rightarrow \mathrm{T}\right)$ in PcvelA gene of NCPC10086 causes glutamine $^{315}$ to stop codon (Q315Stop). Through functional domain scanning, we can see that position 28-238 is velvet factor non-mutated.

\section{Discussion}

We sequenced the whole genome of an industal highpenicillin producing strain NCPC10086 and provided an integral whole geome comparison results with Wisconsin54-1255. A total genome size of $32.3 \mathrm{Mb}$ was assembled with contig N50 of $661 \mathrm{~Kb}$ and scaffold N50 of $2.8 \mathrm{Mb}$. The gene structures were predicted with a combined de novo and homology-based approach, and annotated by four gene annotation systems.

By whole genome comparative analysis, we observed a large number of mutations, insertions and deletions, and structural variations. There are 69 "new" genes that not exist in the genome sequence of Wisconsin54-1255 and some of them are involved in energy metabolism, nitrogen metabolism and glutathione metabolism. As was expected, the high-penicillin producing strain needs more energy for penicillin synthesis, sorting, transport and processing, and we confirm some new genes participate in it. One "new" gene was discovered in nitrogen metabolism, which is up regulated strongly in cultures supplemented with the side chain precursor PAA (phenylacetic acid) in high-producing strain [36]. Both cysteine biosynthesis and the oxidized glutathione need NADPH, if glutathione metabolism is more active, NADPH could be reserved for more cysteine biosynthesis to improve the penicillin production. Our "new" gene involved in glutathione metabolism may impact on this process.

The penicillin biosynthetic genes cluster (PBC) is the well-known dominant core for penicillin production existing in all strains; copy number and fragment arrangement are the key features for $\mathrm{PBC}$. The high-penicillin producing strain, NCPC10086, has seven copies of PBC and one $53.7 \mathrm{~Kb}$ "new shift fragment" with unique arrangement 


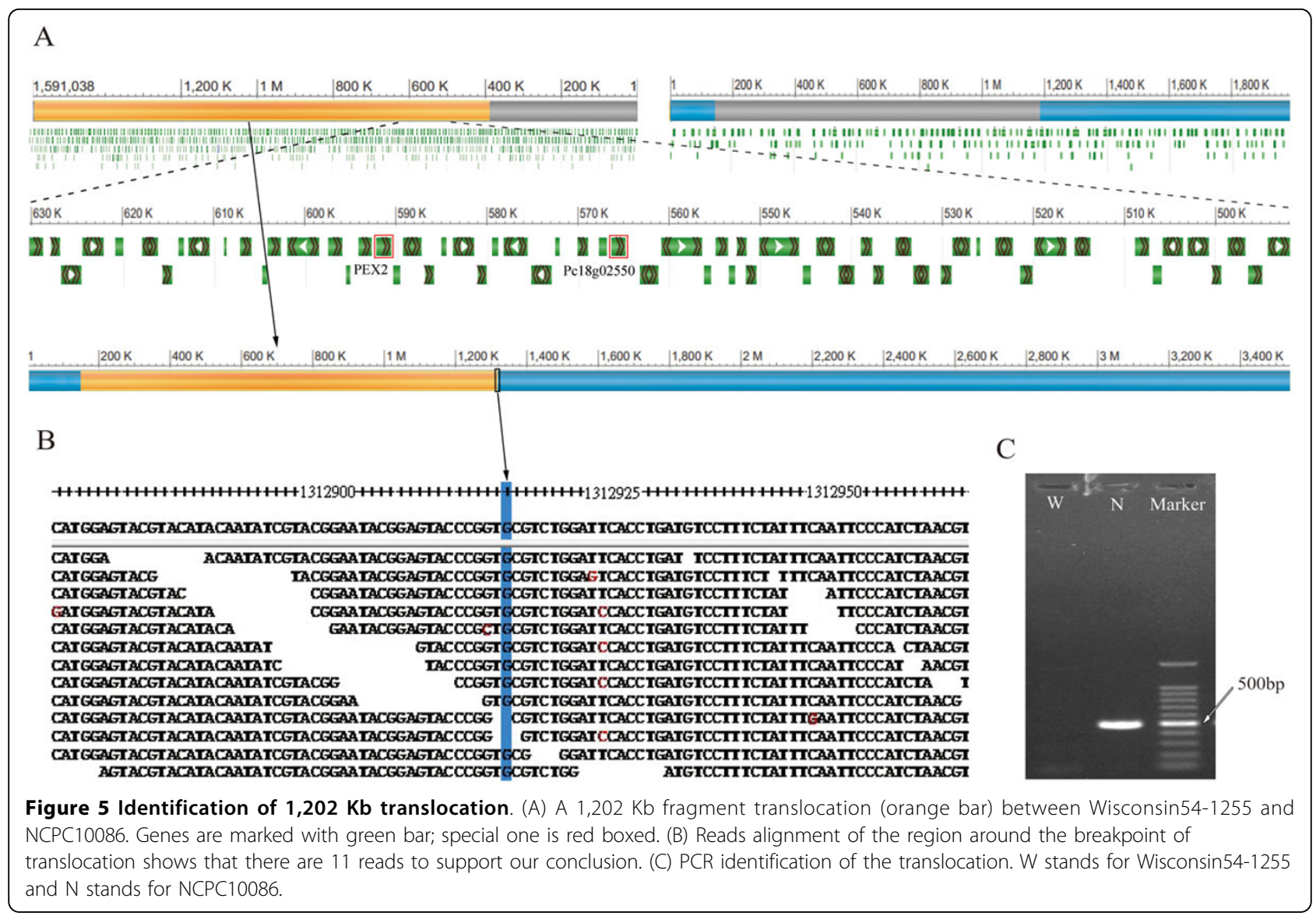

type. The TTTACA sequence and its inverse complement TGTAAA sequence could be hotspots for site-specific recombination after multiple mutations. This process may aim to repair damage from mutations by nitrosoguanidine. We found two large translocations in NCPC10086; one is a $266 \mathrm{~Kb}$ fragment in subtelomere transferred to centromere including genes regulating nitrogen metabolite repression; another is a 1,202 Kb fragment consists of a mitochondrial ADP/ATP carrier involved in energy metabolism and peroxin-2 gene involved in peroxysome pathway.
Due to our comparative genomics statistics results, we predicted that energy metabolism and nitrogen metabolism plays an important role in penicillin production together with glutathione metabolism and peroxysome pathway. To further analysis genes involved in those processes, we looked into two types of genes deeper, pahA gene set and velvet-like complex genes. Translocation, stop codon mutation, synonymous and non-synonymous mutations are found there. These variations may impact the homogentisate pathway for PAA catabolism as well as

Table 4 Single nuclear variations (SNVs) involved in homogentisate pathway and the regulators of penicillin biosynthesis

\begin{tabular}{|c|c|c|c|}
\hline Gene & $\begin{array}{l}\text { Length } \\
\text { (bp) }\end{array}$ & Description & $\begin{array}{l}\text { Discrepancies } \\
\quad \text { (SNVs) }\end{array}$ \\
\hline pahA & 1,727 & $\begin{array}{l}\text { A phenylacetate 2-hydroxylase which catalyzes the first step of the homogentisate pathway for PAA } \\
\text { catabolism }\end{array}$ & - \\
\hline pahB & 1,797 & Strongly similar to pahA & - \\
\hline pahC & 1,785 & Strongly similar to pahA & $\begin{array}{l}\text { non-synonymous } \\
\text { (G482C) }\end{array}$ \\
\hline pahD & 2,423 & Strongly similar to pahA & $\begin{array}{l}\text { synonymous } \\
(\text { C1976T) }\end{array}$ \\
\hline PclaeA & 1,340 & A global regulator of secondary metabolism & - \\
\hline PcvelA & 1,745 & An activator and repressor of secondary metabolism & $\begin{array}{l}\text { non-synonymous } \\
\quad(\text { C1002T) }\end{array}$ \\
\hline
\end{tabular}


global regulation of secondary metabolism, including penicillin biosynthesis, sporulation and pigmentation.

We found out many mutations and structural variations, but how many of them and how they affect the penicillin yield is still a formidable challenge. Efficient approaches to narrow down the possibilities are to sequence more genomes for common variations and system biological investigation using "omic" data [45]. Through genome resequencing and functional analysis, identification of precise mutations in strains with altered phenotypes will add insight into specific gene functions and guide further metabolic engineering efforts.

\section{Conclusions}

This is the first high-quality genome of high-penicillin producing industrial stain of Penicillium chrysogenum, which can provides abundant genetic information for broad biomedical researchers. Through comparative genomics analysis with low-producing strain, we found a lot of mutations, insertions and deletions, and structural variations. Moreover, we showed some "new" genes not existent in the public genome sequence of Wisconsin54-1255 involved in energy metabolism, nitrogen metabolism and glutathione metabolism. Most remarkably, for the penicillin biosynthesis cluster, we are surprised to find a $53.7 \mathrm{~Kb}$ new "shift fragment" in our high-producing strain and the type of fragment arrangement is unique. In addition, we addressed a $266 \mathrm{~Kb}$ translocation including a regulator of nitrogen metabolite repression and a 1,202 Kb translocation including genes involved in energy metabolism and peroxysome pathway. Our findings lay a foundation for the insights into the high-penicillin producing mechanism and metabolic engineering in the future.

\section{Methods}

\section{Source of sample and culture conditions}

P. chrysogenum NCPC10086 strain was selected for genome sequencing as it was commercialized in North China Pharmaceutical Group Corporation. Spore suspensions of NCPC10086 were inoculated in $40 \mathrm{~mL}$ of seed medium (20 g/L sucrose corn steep liquor, $20 \mathrm{~g} / \mathrm{L}$ sucrose, $5 \mathrm{~g} / \mathrm{L}$ yeast extract, $5 \mathrm{~g} / \mathrm{L} \mathrm{CaCO}_{3}$, pH 5.8) in $250 \mathrm{ml}$ flasks and incubated on a rotary shaker (250 r.p.m.) at $26^{\circ} \mathrm{C}$ for $24 \mathrm{~h}$. Two milliliters of seed culture were transferred to $40 \mathrm{~mL}$ of fermentation medium (35 g/L lactose, $30 \mathrm{~g} / \mathrm{L}$ corn steep liquor, $5 \mathrm{~g} / \mathrm{L}(\mathrm{NH} 4)_{2} \mathrm{SO} 4,1 \mathrm{~g} / \mathrm{L} \mathrm{KH}_{2} \mathrm{PO}_{4}, 1 \mathrm{~g} / \mathrm{L}, \mathrm{K}_{2} \mathrm{SO}_{4}$, $10 \mathrm{~g} / \mathrm{L} \mathrm{CaCO}_{3}, 2 \mathrm{~g} / \mathrm{L}$ phenylacetic acid, $6 \mathrm{ml} / \mathrm{L}$ corn oil, $\mathrm{pH}$ 6.0) and grown at $26^{\circ} \mathrm{C}$ with shaking at 250 r.p.m.

\section{Genome sequencing and assembly}

The genome of $P$. chrysogenum NCPC10086 strain was sequenced by whole genome random sequencing method $[20,21]$. We used Roche 454 GS FLX system to produce $18 \times$ coverage single-end reads with an average read length of $410 \mathrm{bp}$ to do contig assembly. Moreover, 3-4 Kb and 6-8 $\mathrm{Kb}$ mate-pair libraries were produced to do contig and scaffold assembly, with $5 \times$ coverage sequenced by ABI 3730 system and $1 \times$ coverage sequenced by Megabace1000 system. ABI 3730 and Megabace1000 produced an average read length of 659 bp and 739 bp respectively. Phred and Phrap [46,47] were used to deal with the raw data from ABI 3730 and Megabace1000. Hybrid assembly was performed using Newbler [24] with all single-end and mate-pair reads by overlap-layout-consensus strategy. After assembly, we aligned our contigs to the reference sequence [12] to predict the gaps sizes distribution in our genome. According to the gaps size distribution, we designed 1-2 Kb mate-pair inserted fragment library to do scaffolding. We mapped the mate-pair high quality reads onto the scaffolds and used the reads to fill the gaps if one of the mate-pair reads located at the edge of gaps. At last, redundant sequences were deleted through self-alignment.

\section{Gene prediction and annotation}

The repeat sequences of $P$. chrysogenum NCPC10086 were masked throughout the genome using RepeatMasker (v 3.3.0) and the RepBase library (20120418) [48] with Tandem Repeats Finder (v 3.2.1)[49]. The gene structures were predicted with a combined de novo and homologybased approach. Firstly, for all repeat-masked scaffolds larger than $1 \mathrm{~Kb}$, Fgenesh (v 3.1.2)[28] and GeneMark-ES (v2.3e)[29] were performed on the whole genomic sequence to provide an initial set of predicted ORFs. Fgenesh is trained on sequences of Penicillium funiculosum and GeneMark-ES is upon the self-training algorithm for fungal genomes. Preference was given to Fgenesh genes, and all predicted protein should be larger than 10aa. Secondly, we use the gene prediction results of $P$. chrysogenum Wisconsin54-1255 to revise and complement our predicted genes by homology searching. At last, the former two results were integrated together as predicted genes. All predicted proteins were blastp [50] against databases of GenBank's non-redundant proteins, InterProscan [30], Swiss-Prot/UniProtKB [32] and Gene Ontology (GO), and the best alignment of every protein was considered its annotation. No alignment results by blastp of predicted proteins were automatically considered as hypothetical proteins. We presented unique and shared proteins among four gene annotation systems by venn diagram (http://bioinfogp.cnb.csic.es/tools/venny/index.html). WEGO [51] was used to plot GO annotation results. The pathway analysis is carried out by KAAS (v 1.67x) with SBH method [52].

\section{Calling single nuclear variations (SNVs), insertions and deletions (InDels) and copy number variation (CNVs)}

Based on the assembled P. chrysogenum Wisconsin54-1255, we realigned all of the high-quality reads with the genome 
by SOAP(v 2.21) [53] to identify the SNVs. In the reads gap-free alignment process, at most two mismatches were allowed between a read and the reference, and best hits were selected. Multiple reads mapping results were filtered. We use SOAPsnp (v1.03)[54], a statistical model based on Bayesian theory and Illumina quality system, to calculate a probability for each possible genotype at each position on the reference genome. We used five thresholds to filter out unreliable SNV results: (1) we required at least five reads for each SNV; (2) average quality of each SNV had to be higher than 20; (3) the overall depth had to be less than 150 ; (4) the approximate copy number of flanking sequences had to be less than 2 (to avoid misreading SNV caused by the alignment of similar reads from repeat units or by copy number variations); (5) there had to be at least one pair of mate-pair reads to support. For InDel detection, we use Pindel(v0.2.4) [55] to find breakpoints of large deletions and medium-sized insertions from paired-end or single reads. The short reads alignment is by BWA- backtrack (v0.5.9) [56] and long reads is by BWA-SW [57]. We use SAM Tools(v0.1.17) [58] to manipulate alignments in the SAM format. Copy number variations detection is by CNV-seq [59] which is based on a robust statistical model with 50x high-quality reads from Illumina HiSeq 2000 .

\section{Identification of structural variations}

We used Blat (v 34) [60] with default parameter to align scaffolds of $P$. chrysogenum NCPC10086 to the reference, whole genome of $P$. chrysogenum Wisconsin54-1255, to search colinearity between them. The alignment results of each scaffold indicate a candidate location of the scaffold. For scaffolds with multiple hits, the top ten hits with highest sequence similarity remained as candidate locations. The alignment with the longest matches in a linear orientation between a scaffold and the reference was picked as 'best-hit' of the scaffold. After finding structural variations, we use Blastn with parameter ' $1 \mathrm{e}^{-5}$ ' to check the detail alignment results. We randomly pick up $20 \times$ mate-pair reads from $180 \times$ high-quality Illumina HiSeq 2000 reads. Reads mapping is by SOAP ( $\mathrm{v}$ 2.21) [53] and the alignment result is visualized by MapView (v 3.4.1) [61]. The 5'-3' primers of PCR identification of structural variations of "266 Kb translocation" are ACCTGGCGTGCCTCATGCAGCG and TTGGGGTGGAATGACGTGGGG, which are before $200 \mathrm{bp}$ and after $300 \mathrm{bp}$ of the breakpoint. The 5'-3' primers of PCR identification of structural variations of "1,202 Kb translocation" are ACCTGTGGGGATCATTAGCCTCC and ACTCGGATAGTCTAGGTTCGGCGG, which are before $250 \mathrm{bp}$ and after $220 \mathrm{bp}$ of the breakpoint.

\section{Availability of supporting data}

P. chrysogenum strain NCPC10086 genome sequences are available via GenBank/EMBL/DDBJ under the accession APKG00000000.

\section{Additional material}

Additional file 1: The list of polymorphic genes with nonsynonymous mutations.

\section{Competing interests}

The authors declare that they have no competing interests.

\section{Authors' contributions}

$J Z$ performed all the bioinformatic analyses and drafted the manuscript. $Y Z$ $J L, M D, G Z$ and $L Z$ performed experiments. JZ and FW coordinated the work. JX, JY, JW, BD all contributed to the conception and design of the study. JW revised the manuscript. All authors read and approved the final manuscript.

\section{Declaration}

Publication of this article was funded by grant from the Natural Science Foundation of China (31271386 and 31101063), grant (2012AA020409) from the National Programs for High Technology Research and Development (863 Program), the Ministry of Science and Technology of the People's Republic of China.

This article has been published as part of BMC Genomics Volume 15 Supplement 1, 2014: Selected articles from the Twelfth Asia Pacific Bioinformatics Conference (APBC 2014): Genomics. The full contents of the supplement are available online at http://www.biomedcentral.com/ bmcgenomics/supplements/15/S1.

\section{Authors' details}

${ }^{1}$ New Drug Research and Development Center of North China Pharmaceutical Group Corporation, National Engineering Research Center of Microbial Medicine, Hebei Industry Microbial Metabolic Engineering \& Technology Research Center, Shijiazhuang, Hebei 050015, China. ${ }^{2}$ CAS Key Laboratory of Genome Sciences and Information, Beijing Institute of Genomics, Chinese Academy of Sciences, Beijing 100101, China. ${ }^{3}$ University of Chinese Academy of Sciences, Beijing 100049, China.

Published: 24 January 2014

\section{References}

1. Drews J: Drug Discovery: A Historical Perspective. Science 2000, 287:1960-1964.

2. Hamed RB, Gomez-Castellanos JR, Henry L, Ducho C, McDonough MA, Schofield CJ: The enzymes of beta-lactam biosynthesis. Nat Prod Rep 2012, 30:21-107.

3. Fleming A: On the Antibacterial Action of Cultures of a Penicillium, with Special Reference to their Use in the Isolation of B. influenzæ. British Journal of Experimental Pathology 1929, 10:226-236.

4. Schofield CJ, Baldwin JE, Byford MF, Clifton I, Hajdu J, Hensgens C, Roach P: Proteins of the penicillin biosynthesis pathway. Curr Opin Struct Biol 1997, 7:857-864.

5. Brakhage AA: Molecular regulation of beta-lactam biosynthesis in filamentous fungi. Microbiol Mol Biol Rev 1998, 62:547-585.

6. Brakhage AA, Sprote P, Al-Abdallah Q, Gehrke A, Plattner H, Tuncher A: Regulation of penicillin biosynthesis in filamentous fungi. Adv Biochem Eng Biotechnol 2004, 88:45-90.

7. Barredo JL, Diez B, Alvarez E, Martin JF: Large amplification of a 35-kb DNA fragment carrying two penicillin biosynthetic genes in high penicillin producing strains of Penicillium chrysogenum. Curr Genet 1989, 16:453-459.

8. van den Berg MA, Westerlaken I, Leeflang C, Kerkman R, Bovenberg RAL: Functional characterization of the penicillin biosynthetic gene cluster of Penicillium chrysogenum Wisconsin54-1255. Fungal Genetics and Biology 2007, 44:830-844.

9. Fierro F, Garcia-Estrada C, Castillo NI, Rodriguez R, Velasco-Conde T, Martin JF: Transcriptional and bioinformatic analysis of the $56.8 \mathrm{~kb}$ DNA region amplified in tandem repeats containing the penicillin gene cluster in Penicillium chrysogenum. Fungal Genetics and Biology 2006, 43:618-629. 
10. Cullen D: The genome of an industrial workhorse. Nature Biotechnology 2007, 25:189-190.

11. van den Berg MA: Impact of the Penicillium chrysogenum genome on industrial production of metabolites. Appl Microbiol Biotechnol 2011, 92:45-53.

12. van den Berg MA, Albang R, Albermann K, Badger JH, Daran JM, Driessen AJM, Garcia-Estrada C, Fedorova ND, Harris DM, Heijne WHM, et al: Genome sequencing and analysis of the filamentous fungus Penicillium chrysogenum. Nature Biotechnology 2008, 26:1161-1168,

13. Fierro F, Barredo JL, Diez B, Gutierrez S, Fernandez FJ, Martin JF: The penicillin gene cluster is amplified in tandem repeats linked by conserved hexanucleotide sequences. Proceedings of the National Academy of Sciences of the United States of America 1995, 92:6200-6204.

14. Kiel JA, van der Klei IJ, van den Berg MA, Bovenberg RA, Veenhuis M: Overproduction of a single protein, Pc-Pex11p, results in 2-fold enhanced penicillin production by Penicillium chrysogenum. Fungal Genetics and Biology 2005, 42:154-164.

15. Jami MS, Barreiro C, Garcia-Estrada C, Martin JF: Proteome analysis of the penicillin producer Penicillium chrysogenum: Characterization of protein changes during the industrial strain improvement. Mol Cell Proteomics 2010.

16. Andersen MR, Salazar MP, Schaap PJ, van de Vondervoort PJ, Culley D, Thykaer J, Frisvad JC, Nielsen KF, Albang R, Albermann K, et al: Comparative genomics of citric-acid-producing Aspergillus niger ATCC 1015 versus enzyme-producing CBS 513.88. Genome Res 2011, 21:885-897.

17. Wohlbach DJ, Kuo A, Sato TK, Potts KM, Salamov AA, Labutti KM, Sun $H$ Clum A, Pangilinan $\mathrm{JL}$, Lindquist $E A$, et al: Comparative genomics of xylose-fermenting fungi for enhanced biofuel production. Proc Natl Acad Sci USA 2011, 108:13212-13217.

18. Cornell MJ, Alam I, Soanes DM, Wong HM, Hedeler C, Paton NW, Rattray M, Hubbard SJ, Talbot NJ, Oliver SG: Comparative genome analysis across a kingdom of eukaryotic organisms: specialization and diversification in the fungi. Genome Res 2007, 17:1809-1822.

19. Kellis M, Patterson N, Endrizzi M, Birren B, Lander ES: Sequencing and comparison of yeast species to identify genes and regulatory elements. Nature 2003, 423:241-254.

20. Weber $\mathrm{L}$, Myers EW: Human whole-genome shotgun sequencing. Genome Res 1997, 7:401-409.

21. Fleischmann RD, Adams MD, White O, Clayton RA, Kirkness EF, Kerlavage AR, Bult CJ, Tomb JF, Dougherty BA, Merrick JM, et al: Wholegenome random sequencing and assembly of Haemophilus influenzae Rd. Science 1995, 269:496-512.

22. Shendure J, Ji H: Next-generation DNA sequencing. Nat Biotechnol 2008, 26:1135-1145.

23. MacLean D, Jones JDG, Studholme DJ: Application of 'next-generation' sequencing technologies to microbial genetics. Nature Reviews Microbiology 2009.

24. Margulies M, Egholm M, Altman WE, Attiya S, Bader JS, Bemben LA, Berka J, Braverman MS, Chen YJ, Chen Z, et al: Genome sequencing in microfabricated high-density picolitre reactors. Nature 2005, 437:376-380.

25. Sanger F, Nicklen $S$, Coulson AR: DNA sequencing with chain-terminating inhibitors. Proc Natl Acad Sci USA 1977, 74:5463-5467.

26. Fedurco M, Romieu A, Williams $S$, Lawrence I, Turcatti G: BTA, a novel reagent for DNA attachment on glass and efficient generation of solidphase amplified DNA colonies. Nucleic Acids Res 2006, 34:e22.

27. Turcatti G, Romieu A, Fedurco M, Tairi AP: A new class of cleavable fluorescent nucleotides: synthesis and optimization as reversible terminators for DNA sequencing by synthesis. Nucleic Acids Res 2008, 36:e25.

28. Salamov AA, Solovyev W: Ab initio gene finding in Drosophila genomic DNA. Genome Res 2000, 10:516-522.

29. Ter-Hovhannisyan V, Lomsadze A, Chernoff YO, Borodovsky MG-E: Gene prediction in novel fungal genomes using an ab initio algorithm with unsupervised training. Genome Research 2008, 18:1979-1990.

30. Quevillon E, Silventoinen V, Pillai S, Harte N, Mulder N, Apweiler R, Lopez R: InterProScan: protein domains identifier. Nucleic Acids Res 2005, 33: W116-120.

31. Zdobnov EM, Apweiler R: InterProScan-an integration platform for the signature-recognition methods in InterPro. Bioinformatics 2001, 17:847-848.

32. Boeckmann B: The SWISS-PROT protein knowledgebase and its supplement TrEMBL in 2003. Nucleic Acids Research 2003, 31:365-370.
33. O'Donovan C, Martin MJ, Gattiker A, Gasteiger E, Bairoch A, Apweiler R: High-quality protein knowledge resource: SWISS-PROT and TrEMBL. Brief Bioinform 2002, 3:275-284.

34. Camon E, Magrane M, Barrell D, Binns D, Fleischmann W, Kersey P, Mulder N, Oinn T, Maslen J, Cox A, Apweiler R: The Gene Ontology Annotation (GOA) project: implementation of GO in SWISS-PROT, TrEMBL, and InterPro. Genome Res 2003, 13:662-672.

35. Ashburner M, Ball CA, Blake JA, Botstein D, Butler H, Cherry JM, Davis AP, Dolinski K, Dwight SS, Eppig JT, et al: Gene ontology: tool for the unification of biology. The Gene Ontology Consortium. Nat Genet 2000, 25:25-29.

36. Harris DM, van der Krogt ZA, Klaassen P, Raamsdonk LM, Hage $S$, van den Berg MA, Bovenberg RA, Pronk JT, Daran JM: Exploring and dissecting genome-wide gene expression responses of Penicillium chrysogenum to phenylacetic acid consumption and penicillin $G$ production. BMC Genomics 2009, 10:75.

37. Nasution U, van Gulik WM, Ras C, Proell A, Heijnen JJ: A metabolome study of the steady-state relation between central metabolism, amino acid biosynthesis and penicillin production in Penicillium chrysogenum. Metab Eng 2008, 10:10-23.

38. Fierro F, Gutierrez S, Diez B, Martin JF: Resolution of four large chromosomes in penicillin-producing filamentous fungi: the penicillin gene cluster is located on chromosome II (9.6 Mb) in Penicillium notatum and chromosome I (10.4 Mb) in Penicillium chrysogenum. Mol Gen Genet 1993, 241:573-578.

39. Raper KB, Alexander DF, Coghill RD: Penicillin: II. Natural Variation and Penicillin Production in Penicillium notatum and Allied Species. J Bacteriol 1944, 48:639-659.

40. Šmidák R, Kralovičová M, Ševčíková B, Jakubčová M, Kormanec J, Timko J, Turňa J: Sequence analysis and gene amplification study of the penicillin biosynthesis gene cluster from different strains of Penicillium chrysogenum. Biologia 2010, 65:1-6

41. Galagan JE, Calvo SE, Cuomo C, Ma LJ, Wortman JR, Batzoglou S, Lee SI, Basturkmen M, Spevak CC, Clutterbuck J, et al: Sequencing of Aspergillus nidulans and comparative analysis with A. fumigatus and A. oryzae. Nature 2005, 438:1105-1115.

42. Rodriguez-Saiz M, Barredo JL, Moreno MA, Fernandez-Canon JM, Penalva MA, Diez B: Reduced function of a phenylacetate-oxidizing cytochrome p450 caused strong genetic improvement in early phylogeny of penicillin-producing strains. J Bacteriol 2001, 183:5465-5471.

43. Kosalkova K, Garcia-Estrada C, Ullan RV, Godio RP, Feltrer R, Teijeira F, Mauriz E, Martin JF: The global regulator LaeA controls penicillin biosynthesis, pigmentation and sporulation, but not roquefortine $C$ synthesis in Penicillium chrysogenum. Biochimie 2009, 91:214-225.

44. Hoff B, Kamerewerd J, Sigl C, Mitterbauer R, Zadra I, Kurnsteiner H, Kuck U: Two components of a velvet-like complex control hyphal morphogenesis, conidiophore development, and penicillin biosynthesis in Penicillium chrysogenum. Eukaryot Cell 2010, 9:1236-1250.

45. Quo CF, Kaddi C, Phan JH, Zollanvari A, Xu M, Wang MD, Alterovitz G: Reverse engineering biomolecular systems using -omic data: challenges, progress and opportunities. Briefings in Bioinformatics 2012, 13:430-445.

46. Ewing B, Hillier L, Wendl MC, Green P: Base-calling of automated sequencer traces using phred. I. Accuracy assessment. Genome Res 1998, 8:175-185.

47. Ewing B, Green P: Base-calling of automated sequencer traces using phred. II. Error probabilities. Genome Res 1998, 8:186-194.

48. Jurka J, Kapitonov W, Pavlicek A, Klonowski P, Kohany O, Walichiewicz J: Repbase Update, a database of eukaryotic repetitive elements. Cytogenet Genome Res 2005, 110:462-467.

49. Benson G: Tandem repeats finder: a program to analyze DNA sequences. Nucleic Acids Res 1999, 27:573-580.

50. Altschul SF, Gish W, Miller W, Myers EW, Lipman DJ: Basic local alignment search tool. J Mol Biol 1990, 215:403-410.

51. Ye J, Fang L, Zheng H, Zhang Y, Chen J, Zhang Z, Wang J, Li S, Li R, Bolund L: WEGO: a web tool for plotting GO annotations. Nucleic Acids Res 2006, 34:W293-297.

52. Moriya Y, Itoh M, Okuda S, Yoshizawa AC, Kanehisa M: KAAS: an automatic genome annotation and pathway reconstruction server. Nucleic Acids Res 2007, 35:W182-185

53. Li R, Li Y, Kristiansen K, Wang J: SOAP: short oligonucleotide alignment program. Bioinformatics 2008, 24:713-714. 
54. Li R, Li Y, Fang X, Yang H, Wang J, Kristiansen K: SNP detection for massively parallel whole-genome resequencing. Genome Res 2009, 19:1124-1132.

55. Ye K, Schulz MH, Long Q, Apweiler R, Ning Z: Pindel: a pattern growth approach to detect break points of large deletions and medium sized insertions from paired-end short reads. Bioinformatics 2009, 25:2865-2871.

56. Li H, Durbin R: Fast and accurate short read alignment with BurrowsWheeler transform. Bioinformatics 2009, 25:1754-1760.

57. Li H, Durbin R: Fast and accurate long-read alignment with BurrowsWheeler transform. Bioinformatics 2010, 26:589-595.

58. Li H, Handsaker B, Wysoker A, Fennell T, Ruan J, Homer N, Marth G, Abecasis G, Durbin R: The Sequence Alignment/Map format and SAMtools. Bioinformatics 2009, 25:2078-2079.

59. Xie C, Tammi MT: CNV-seq, a new method to detect copy number variation using high-throughput sequencing. BMC Bioinformatics 2009, $10: 80$

60. Kent WJ: BLAT-the BLAST-like alignment tool. Genome Res 2002, 12:656-664.

61. Bao H, Guo H, Wang J, Zhou R, Lu X, Shi S: MapView: visualization of short reads alignment on a desktop computer. Bioinformatics 2009, 25:1554-1555.

doi:10.1186/1471-2164-15-S1-S11

Cite this article as: Wang et al:: Genome sequencing of high-penicillin producing industrial strain of Penicillium chrysogenum. BMC Genomics 2014 15(Suppl 1):S11.

\section{Submit your next manuscript to BioMed Central} and take full advantage of:

- Convenient online submission

- Thorough peer review

- No space constraints or color figure charges

- Immediate publication on acceptance

- Inclusion in PubMed, CAS, Scopus and Google Scholar

- Research which is freely available for redistribution

Submit your manuscript at www.biomedcentral.com/submit
C Biomed Central 